\title{
CONVERSACIONES SOBRE LA TERAPIA NARRATIVA EN PERSPECTIVA NARRATIVA: PASADO, PRESENTE Y FUTURO
}

\section{CONVERSATIONS ABOUT NARRATIVE THERAPY IN NARRATIVE PERSPECTIVE: PAST, PRESENT AND FUTURE}

\author{
Carlos Alexis Chimpén-López \\ Facultad de Enfermería y Terapia Ocupacional, Universidad de Extremadura. Cáceres, España \\ ORCID: https://orcid.org/0000-0001-9384-3960
}

Meritxell Pacheco

Facultat de Psicologia, Ciències de l’Educació i de l’Esport Blanquerna, Universitat Ramon Llull. Barcelona. España

ORCID: https://orcid.org/0000-0003-1665-1425

Cómo referenciar este artículo/How to reference this article:

Chimpén-López, C. A. y Pacheco, M. (2019). Conversaciones sobre la Terapia Narrativa en Perspectiva Narrativa: Pasado, Presente y Futuro. Revista de Psicoterapia, 30(114), 5-16. https://doi.org/10.33898/ rdp.v30i114.317

\section{Resumen}

Este trabajo es la transcripción de una entrevista realizada por Meritxell Pacheco, coordinadora del grupo de trabajo del Col-legi Oficial de Psicòlegs de Catalunya, COPC: La Narrativa en Educació i en Psicoteràpia, a Carlos Chimpén, presidente de AETEN, Asociación Española de Terapia Narrativa. La entrevista se desarrolla en el contexto de la jornada El Matí Narratiu, celebrada en la sede del COPC y permite reflexionar sobre la terapia narrativa en el momento actual, comprendiendo sus origenes, y anticipando posibles desarrollos futuros. También se conversa sobre las posibilidades de formación de terapeutas noveles y sobre como es necesario que a medida que se gana en experiencia se deconstruyan los mapas de la práctica narrativa de Michael White y se recupere el espiritu de aventura de la terapia narrativa.

Palabras Clave: psicoterapia narrativa, técnicas terapéuticas, mapas de la práctica narrativa, conversación, co-construcción.

\begin{abstract}
This work is the transcript of an interview conducted by Meritxell Pacheco, coordinator of the working group of the Col-legi Oficial de Psicolegs de Catalunya, COPC: La Narrativa en Educació i en Psicoteràpia, to Carlos Chimpén, president of AETEN, Asociación Española de Terapia Narrativa. The interview takes place in the context of the Matí Narratiu conference, held at COPC headquarter, and allows us to reflect on narrative therapy at the present time, understanding its origins, and anticipating possible future developments. It is also talked about the possibilities of training for new therapists and how it is necessary that as someone gains experience, maps of Michael White's narrative practice are deconstructed and the adventure spirit of narrative therapy could be restored.

Keywords: narrative psychotherapy, therapeutic techniques, maps of narrative practice, conversation, co-construction.
\end{abstract}

Fecha de recepción: 15 de julio de 2019. Fecha de aceptación: 16 de septiembre de 2019.

Correspondencia sobre este artículo:

E-mail: cchimpen@unex.es

Dirección postal: Carlos Alexis Chimpén, Avda. de la Universidad s/n, 10002, Cáceres.

(C) 2019 Revista de Psicoterapia 
Meritxell(M): Conversamos con el Dr. Carlos Chimpén, presidente deAETEN, Asociación Española de Terapia Narrativa, en el contexto de la jornada El Matí Narratiu, organizada en el Col-legi Oficial de Psicologia de Catalunya, COPC, por el grupo de trabajo: La Narrativa en Educació i en Psicoteràpia que tengo el placer coordinar. El Dr. Chimpén acaba de participar con la conferencia titulada Terapia Narrativa: Co-Construyendo el Futuro de Nuestras disciplinas, en la que nos ha transmitido lo que él denomina "el espíritu de la aventura narrativa” y que consiste en dejarse llevar por las posibilidades de co-creación que se abren en cada nueva conversación con un consultante y contribuir a que, como él mismo dice, surja la magia.

Explícanos, Carlos, ¿cómo entiendes tú la aventura narrativa?

Carlos (C): La aventura narrativa es comprender que la persona o personas que tienes delante van a crear ciertos contextos y conversaciones nuevos, y tú tienes que ser creativo y saber co-construir nuevas historias con esas personas. Eso es la co-creación de una terapia, de una nueva conversación, de una nueva identidad en una cultura concreta. Entonces, incluso podríamos cuestionar y deconstruir, y no hay ningún tipo de problema en hacerlo, los libros de White y Epston y empezar a aplicarlos a nuestra cultura. Porque hay veces que en sus libros aparecen preguntas súper enrevesadas traducidas del inglés, a veces un poco regular, y que cuando las quieres replicar no te salen igual, por supuesto, y a lo mejor ni siquiera encajan. Y es como tiene que ser, porque no tenemos que replicar exactamente sus propuestas originales. Tenemos que adaptar la intervención a quienes somos y al contexto en el que trabajamos. Así es como la aventura continua.

M- La aventura continua. ¿Cómo hacer entonces para que el espíritu de aventura de la terapia narrativa permanezca?

C- Bueno, aquí tenemos un papel importante desde las universidades y escuelas de formación. Tenemos el master en terapia narrativa de la Universidad de Extremadura, el título de experto que va a empezar en Blanquerna, la Asociación Española de Terapia Narrativa, y cada una de las personas que vienen a visitarnos, nuestros clientes, o consultantes, que también nos dan mucho juego para intentar innovar y hacer cosas diferentes. Entonces el espíritu de la narrativa viene de la cocreación relacional con las personas que nos vienen a ver y de la comunidad, como por ejemplo esta comunidad que ha venido hoy a participar en El Matí Narratiu. Eso tiene que seguir vivo, esto es el espíritu de la narrativa.

M- Nos has ofrecido las frases de David Epston, en la plenaria de San Diego de 2018, con motivo de la conmemoración del décimo aniversario de la muerte de Michael White. Nos contabas como Epston llamaba a deconstruir los mapas y a recuperar el espíritu de aventura de la terapia narrativa.

C- Sí, nadie como David sabe explicarlo. Según él, el espíritu de la terapia narrativa conlleva todo esto: "Entusiasmo, irreverencia, improvisación, imaginación, justa indignación frente a la injusticia, solidaridad con los que sufren, creatividad colectiva y una fascinación por el misterio y la magia en el corazón de la vida co- 
tidiana". Y es por eso que, no sé si es justo o adecuado, los terapeutas se sometan a un libro como el de mapas de la práctica narrativa, que se sometan a aprender una determinada forma de hacer conversaciones y seguir replicando. Si hacemos eso nos perdemos el espíritu de la terapia narrativa.

M- Aunque decías también que los terapeutas noveles necesitan esos mapas.

C- Sí, por supuesto. Es cierto que cuando hacemos ciertas intervenciones o preguntas, el terapeuta dice: ¡quiero que digas como se hace eso!, pero cuando uno ya no es tan novel necesita tener este tipo de cuestiones que van mucho más allá de los mapas. Pero permíteme que me quede en la parte de la magia, en el corazón de la vida cotidiana, $\mathrm{y}$ la magia que se crea cuando haces cierto tipo de conversaciones alejándote de lo que es una técnica.

M- Carlos, sobre la magia y el espíritu de la narrativa, me ha gustado mucho la frase que has compartido de Bill O’Hanlon (1994, p.28) y que es justo lo que a veces intentamos transmitir a nuestros estudiantes, no siempre con éxito: "He de dar un aviso, si utilizas la externalización sólo como una técnica probablemente no producirá efectos profundos. Si no crees desde lo profundo de tu alma que la gente no son sus problemas y que sus dificultades son construcciones sociales y personales, entonces no verás estas transformaciones. Cuando Epston o White entran en acción puedes decir que están absolutamente convencidos de que la gente no son sus problemas. Sus voces, sus posturas, todo su ser irradia posibilidad y esperanza”. Importantísimo. ¿Qué necesitamos para que los estudiantes se imbuyan del espíritu de la narrativa? ¿Qué necesitamos para que no sigan sin más los pasos de una conversación externalizadora? ¿Para que salgan del molde y sepan crear magia en las sesiones?

C- Ciertamente, cuando alguien aprende terapia narrativa, si lo que quiere es aplicar un conjunto de técnicas sobre cómo acercarse a la persona, está perdiendo la magia, está perdiendo este tipo de posibilidades. Es decir, la terapia narrativa no es un conjunto de técnicas. La terapia narrativa es una filosofía de vida y una cosmovisión y esto es lo que tienen que interiorizar los estudiantes. Es una filosofía y la filosofía conlleva ciertas estructuras o cierto posicionamiento, como el que ha venido a erigirse como lema de la terapia narrativa: "la persona es la persona, la persona no es el problema” (White, 1984). Pero eso lo llevas hasta el hecho de verlo, cotidianamente, en el día a día. No es algo que tiene que ver con la posición de terapeuta donde pongo una disposición especial para ver a la persona lejos de su problema. No es una realidad de tu día a día, es una filosofía de vida.

M- Acaba derivando en una actitud vital. Justamente es uno de los aspectos que más me atraen de la terapia narrativa. El trabajo desde la persona del terapeuta, desde las creencias más nucleares sobre la persona, el bienestar y el conocimiento, no como un rol adquirido, sino como una forma de hacer que surge genuinamente del ser.

C- Exacto, es una actitud, una filosofía de vida. Tú la llevas contigo, y como la llevas contigo cuando te paras delante de la gente sale, no tienes que forzarla. 
Y sale automáticamente, y sale de diferentes formas. No sale siempre de la misma manera, como replicando una técnica, porque no es una técnica. Esto a lo mejor puede conllevar un cierto problema porque a veces las preguntas que surgen, en quienes se acercan a la terapia narrativa, son ¿y cómo hacemos esto? ¿o cómo me sale? ¿O cómo lo puedo adquirir o adherirme a esto? Porque aprender una técnica es fácil, pero luego ¿cómo hago para que se vea lo que yo digo y para tener una postura filosófica delante de alguien? Esa es la pregunta que podríamos hacernos. Y la respuesta es que tendré que empezar a internalizar ciertas cosmovisiones que se promueven desde las prácticas narrativas para que yo pueda hacer eso cuando estoy delante de alguien. Y esa internalización implica: leer, estudiar y como decía Michael White: práctica, práctica y más práctica.

M- No es fácil encontrar el equilibrio entre el aprendizaje de las distintas formas de conversación y practicar, practicar y practicar y, simultáneamente, saber dejarse llevar por la magia de una conversación e innovar.

C- Bueno, es necesario encontrar este equilibrio porque en realidad te salen ciertas cosas cuando ya sabes algo; si no sabes algo no te pueden salir las improvisaciones. Entonces, los mapas pueden estar muy bien para una etapa inicial acerca de cómo hago ciertas conversaciones de la terapia narrativa, pero una vez ya lo he hecho tengo que empezar a adquirir cierta cosmovisión para que cuando me ponga delante de un colegio, de una comunidad, de un profesor, de una queja, de lo que sea, ya me salga esta otra disposición. Cuando se escucha de una forma diferente es porque has asimilado una cierta forma de ver al otro de una forma diferente. Entonces, es una cosmovisión, no son técnicas.

M- Está claro. Es aprender los mapas para luego olvidarlos y ejercer una influencia descentrada desde la persona del terapeuta a la persona del consultante, de ese en concreto y no de otro, y con la cosmovisión narrativa de trasfondo.

C- Sí, sería eso Meritxell. Entonces cuando alguien lee el libro de mapas y observa cómo hacer una conversación de externalización con el paso uno, dos, tres y cuatro... está muy bien, puedes hacerlo al principio... pero ¿y después? ¿Qué pasa cuando te encuentras con alguien y el paso 2 te lo has saltado y has ido al 4...? ¿Qué haces entonces? ¿Vas al tres, vuelves al dos...? No puedes hacer este tipo de cosas. Las conversaciones son vivas, son fluidas, las personas son diferentes y me puedo pasar de una a otra conversación de forma libre sin seguir una estructura predeterminada. Entonces esto es una de las cosas que tenemos que tener muy presente. Si la cosmovisión está clara, puedo saltar entre mapas e incluso me los puedo saltar.

M- Es trabajar desde y para la persona, teniendo claro qué proceso intentamos trabajar en esa conversación, sin sentirse esclavo de una forma concreta de proceder.

C- Puedo saber los mapas, pero puedo hacer lo que sea, y no tengo por qué tener una técnica: es una forma de hablar, una forma de conversar. Y esas formas de conversar son las que crean diferentes contextos y sobre todo, diferentes identidades. ¡Cuidado! en la persona que nos consulta y en nosotros como terapeutas. Porque es bidireccional. 
M- La persona del terapeuta. De persona a persona se crea la magia...

C- Sí, es un cuestionamiento de las técnicas. Y entonces es cuando David Epston habla de la magia de las conversaciones. Y yo no sabría muy bien cómo explicar lo de la magia. Lo puedo explicar desde mi postura, desde como lo siento.

M- Nos has ofrecido en la conferencia las palabras de tu compañera Sara explicando la magia que percibe que se crea en tus conversaciones en terapia.

C- Sí, además Sara escribe precioso, muy poético. "La magia co-construida, a ritmo de preguntas abiertas, historias mágicas entretejidas como redes relacionales, que buscan lo extraordinario y enseñan a empoderar”, decía ella.

M- Es justamente eso. Enfatizando la co-construcción y la relación entre las acciones y la identidad. El cambio duradero y significativo es el que se sustenta en el paisaje de la identidad. Me ha gustado como lo expresaban las palabras de Sara: "Es más fácil caminar en paralelo, al compás de un vaivén que zigzaguea entre la identidad y la acción; es más útil si a mi marcha la acompañan tus pisadas... poderoso encantamiento de este encuentro que hace camino al andar”. Ella hace magia hablando de tu magia en las conversaciones.

C- Sí, y yo le di las gracias por ello. Recordemos que con los elogios a veces te pones un poco incómodo. No estamos muy acostumbrados porque en nuestra cultura, no se admite demasiado. En eso somos distintos a los norteamericanos. Y claro, yo estaba medio incómodo a la vez que agradecido, y decidí pedirle que pusiera por escrito lo que me había dicho y por eso he podido compartirlo aquí.

M- ¿Y cómo provocar esa magia con técnicas? ¿Es posible?

C- ¿Cómo hacemos poesía con técnicas? Se puede aprender a escribir en un taller literario, pero una vez que has aprendido tienes que hacer una cosa diferente, y que brote otra cosa diferente. Esa es la magia de la que estamos hablando. Del espíritu de la terapia narrativa. Y eso no se logra replicando lo que hacían White y Epston, o lo que hago yo o lo que hacen otros autores en libros. Tienes que apropiarte de eso. Y tienes que hacer magia con eso. Y el que lo va a decir no eres tú. Lo va a decir la persona que está delante de ti.

M- ¿Y cómo sabemos si esa magia le está siendo útil a la persona que estamos atendiendo? Disfrutamos tanto co-creando conversaciones creativas... ¿cómo evitar el riesgo de ensimismarnos en esa poesía hacia el empoderamiento? Debemos asegurarnos de que está llegando, si no perdería toda su función.

C- Quizás se puede ir a lo más sencillo, haciendo "Exótico lo doméstico" como decía Michael White (1997, p. 30) utilizando las palabras de Bourdieu para objetivar el mundo familiar y dado por sentado. No tengamos miedo de preguntarle: Oye, lo que estamos haciendo, ¿te está sirviendo? Esto de lo que estamos hablando, ¿está bien para ti? ¿quieres que sigamos hablando de esto o quieres que cambiemos y hablemos de otra cosa? ¿te apetece que hablemos de esto que me has dicho de tu madre? ¿te apetece que invitemos a tu madre a la consulta? ¿querrías que hiciéramos ciertas conversaciones con respecto a lo que tu madre puede ver en ti? Y acercarte de una forma respetuosa hacia otras conversaciones que crean 
una identidad alternativa o diferente. Pero no siguiendo técnicas, sino siguiendo la magia que puede surgir de este tipo de conversaciones, de la creatividad, de la improvisación... Sabiendo lo que haces, por supuesto, para eso diseñamos programas de formación específicos en terapia narrativa. Pero una vez aprendido hay que "olvidarlo" y aplicarlo de forma diferente a cada diferente conversación con diferentes personas. Esa es la magia.

M- Ante esta idea me aparece la metáfora del baile. La persona que está aprendiendo a bailar marca los pasos y se asegura de estar haciéndolo correctamente y en el orden preciso. Pero en esos momentos iniciales... eso todavía no tiene apariencia de baile. En el bailarín profesional, sin embargo, no percibes los pasos, percibes la danza... La magia, en este caso a partir del movimiento. Y es capaz de alterar el orden preestablecido de los pasos, crear, innovar, dependiendo de lo que surge en el momento.

C- La metáfora del baile es muy útil para entenderlo. Sí, es algo como lo que propones. Es cuando lo que está sucediendo pasa a formar parte de ti y viceversa. Yo creo que eso es a lo que se estaba refiriendo Sara. La magia del co-crear en harmonía y de sentirse fluir en el momento. Le dije que me sentía orgulloso y agradecido por sus palabras. Porque en el fondo eso es lo que se crea. Es una co-creación: tú me devuelves a mí algo al escuchar tus palabras. Eso también es terapia para mí. Y esto es poner a la otra persona en la posición de persona. Eso es terapia narrativa: somos personas conversando en torno a problemas para crear o co-crear identidades alternativas a las que el problema te puede estar ofreciendo. $\mathrm{Y}$ eso te hace crecer, te hace enriquecer. $\mathrm{Y}$ estas palabras te hacen seguir creando magia y creyendo en las posibilidades.

M- Hablando de la magia de las conversaciones, de cómo disfrutamos cocreando con las personas que vienen a consultarnos, ¡no parece que este sea un trabajo por el que cobramos un dinero, jajajaja!

C- Sí, es otra pregunta que podríamos hacernos: ¿tenemos que alejarnos de ese espíritu de la terapia como un negocio? Porque es verdad que intentas hablar con ciertas personas que se convierten en capos porque escriben muchos libros, y está muy bien escribir libros, crear, pero cuando les invitas a dar una charla te cobran $2.500 €$ por día, hagan o no hagan la formación. Quizás ese no sea el espíritu de la aventura narrativa. No digo que no haya que ganar dinero, porque hay que comer, por supuesto, y hay que valorar el trabajo que estamos haciendo, por supuesto, pero una de las posturas éticas de la terapia narrativa también es el anti-individualismo y el anti-neoliberalismo. Porque estamos viendo que el capitalismo a ultranza nos fuerza a veces a entrar en una rueda de hámster. De seguir trabajando, trabajando y trabajando para generar y, a veces, cuando eres capaz de generar te sientes muy bien, pero cuando no eres capaz de generar empieza la culpa. O empiezan ciertos "amiguitos", como el perfeccionismo, a machacarte.... Y a intentar... bueno... dominar tu vida. Entonces, desde las prácticas narrativas esto también es una aventura: el ¿qué hacer para enfrentarnos a ciertos posicionamientos sociales o culturales que 
oprimen a las personas? Algunos de ellos son el individualismo y la competitividad. M- Entiendo lo que comentas Carlos, sobre todo en cuanto al hecho de cómo nos pueden llegar a oprimir los discursos culturalmente disponibles si les dejamos que ejerzan un rol totalitario, que dominen nuestra vida sin dejar espacio a nada más. Pero el efecto que cada discurso ejerza sobre cada cual, ¿̇no dependerá en gran medida de los significados que cada uno atribuya a estos discursos? Es decir, a modo de ejemplo, tú hablabas del neo-liberalismo asociándolo al individualismo, a la competitividad, a la obsesión por ganar más y más, pero podemos pensar en personas que lo asocian a sentimientos de libertad y que además trabajan en colaboración con otros, quizás apoyando también a comunidades desfavorecidas, y se sienten satisfechos consigo mismos porque con su esfuerzo diario generan trabajo y, por lo tanto, bienestar económico en otras familias. Puedo imaginar y hasta poner cara a estos dos perfiles de posicionamientos neo-liberales, y seguramente puede haber más perfiles. El de la persona dominada por el deseo de incrementar su riqueza por encima de todo, de todos, e incluso del propio bienestar emocional, y que puede llevar también a la explotación laboral de personas con menos posibilidades, y el perfil de neo-liberal que buscando también incrementar beneficios económicos con su trabajo, no se apega de forma rígida a ese deseo y eso le permite disfrutar en su desempeño profesional así como de la satisfacción de generar bienestar económico y más posibilidades laborales también a su alrededor. Tal y como lo veo, no es tanto aproximarse a la cotidianeidad de nuestro trabajo como psicoterapeutas desde un discurso neo-liberal o social-comunista. Podría hacerte también la caricatura de dos perfiles extremos (uno "oprimido y centrado en uno mismo" y otro "liberado y fluyendo con los demás") desde el social- comunismo... Es imposible no posicionarse, a sabiendas o no, en un discurso culturalmente disponible, ¿quizás lo óptimo sería no dejarse dominar, oprimir, por ninguno de ellos? ¿cómo encontramos el termómetro para esto?

C- Una de las cosas que se promueve desde las prácticas narrativas es la deconstrucción de los discursos dominantes que oprimen a las personas. Para ello se plantea el hacer visible lo invisible, el cuestionar lo dado por sentado. Cuando se habla sobre la dominación que pueden ejercer ciertos discursos en las vidas de las personas entonces puedes tomar un posicionamiento con respecto a esos discursos y no dejarte oprimir por ellos. Entonces quizás valga la pena externalizar los efectos de la competitividad y el individualismo en nosotros. Como bien sabes, las conversaciones de externalización es una forma de deconstrucción. Cabe pensar en la pregunta: ¿para qué hago lo que hago? Hago ciertas cosas para destacar o para que nos reconozcan... A lo mejor el reconocimiento, visto desde un ángulo diferente, no es tanto el que se ofrece desde una comunidad científica, sino el que procede de una carta que te manda alguien, el que se produce en una conversación íntima entre dos personas. Eso es un reconocimiento también. Quizás la idea es cuestionar el tipo de cosas que mencionábamos antes. Esa búsqueda de ingresos extremos por una conferencia a lo mejor es una replicación de discursos o modelos 
sociales capitalistas que no van con el espíritu de la terapia narrativa.

M- Está claro que desde el modelo narrativo siempre se ha defendido la colectividad por encima de la individualidad, el co-construir, la generación conjunta de nuevas posibilidades; sus raíces están más en la comunidad que en la individualidad, por supuesto. Pero ¿por qué esto tiene que ser incompatible con unos ingresos económicos determinados o con un modelo de negocio u otro cuando ejercemos? ¿Será más narrativo el terapeuta que trabaje en una cooperativa que quien funde su propia empresa? Entiendo por lo que dices que el que trabaje en la cooperativa estará llevando el espíritu narrativo a la configuración laboral de su trabajo, supongo que te refieres a eso. A ser terapeuta narrativo desde la persona, en todas sus dimensiones, no como un rol o unas técnicas aprendidas. A eso le veo sentido. Y a huir del yugo de los discursos opresores. Pero no creo que sea útil decidir de antemano qué discurso es el más opresor porque posiblemente esto pueda limitar la apertura a nuevos discursos que estén por llegar o que quizás ya están llegando.

C- Las prácticas narrativas no están reñidas con un modelo de negocio u otro, ni tampoco con la riqueza, pero sí se proclama que debemos ser conscientes de las desigualdades, del desequilibrio de poder, de las opresiones de algunas mayorías y las desventajas de las minorías. Se hace política desde la terapia y eso tiene que ver con el posicionamiento del terapeuta y las ideas que el mismo lleva a la sala de terapia. No podemos replicar ciertos modelos de control y poder en nuestra sala de terapia y esto tiene que ver con discursos sociales que se instauran en las personas casi de manera invisible. Esto es lo que se promueve desde la deconstrucción de las prácticas del poder y del saber. Foucault escribió mucho al respecto y White y Epston lo llevaron al terreno de la terapia. Quizás por este motivo la aventura narrativa ha llevado al Dulwich Centre a trabajar en la franja de Gaza y Cisjordania, con gente que ha sido torturada y trabajando con ciertas formas de rescatar los valores o principios o sueños o capacidades de las personas a pesar del trauma vivido. Quizás el hecho de trabajar lo individual teniendo en cuenta los discursos sociales es lo que hace que veamos lo individual desde lo colectivo.

M- Por supuesto.

C- O, por ejemplo, el congreso internacional, promovido por el Dulwich Center será en Rwanda en el 2020. Se hace allí porque hubo un genocidio y se fue a trabajar con víctimas del genocidio. De nuevo para rescatar una historia diferente a la que ofrecía el trauma y no replicar modelos del síndrome de estrés postraumático y el protocolo de cómo tenemos que trabajar con este síndrome, sino rescatar otras historias, presentes en las personas, sin retraumatizar. Ese es el espíritu de la terapia narrativa. No está basado en el negocio, ni en la individualidad, sino en la comunidad, y no está basado en la competitividad, sino en la colaboración. Es un trabajo comunitario. Aunque lo hagas individual es comunitario.

M- Al final es no perder la perspectiva que la persona lo es en su contexto y que ese contexto puede estar oprimiendo y traumatizando, pero también tendrá elementos para la reconstrucción posibilitadora. Nos hablabas también de la terapia 
narrativa como arte, del papel de la creatividad y la imaginación en terapia.

C-Exacto. Cuando ya tienes esa cosmovisión, esa filosofía de trabajo, aparecen otras palabras que también presenta David Epston: el arte, dentro de la terapia. La terapia tiene una parte de aprendizaje, de saber hacer, pero hay otra parte de arte, y cuando estás delante de alguien tiene que salir ese arte. Y sale imaginación, y sale creatividad.

M- Como decías, hay gente que se ve presionada porque dice: “yo no tengo ni imaginación, ni creatividad”.

C- La ventaja de la posición de la terapia narrativa es que la persona que tienes delante sí que tiene arte y creatividad. Es importante no sentirse presionado por la idea de perfeccionismo y de tener que ser creativo sí o sí. Utilizamos la imaginación y la creatividad, ya sean propias o de quienes vienen a consultarnos, para hacer este tipo de entrevistas. Igual que yo hoy en la conferencia he ilustrado esto desde la creatividad de otra persona, de un niño de 10 años.

M- Sí, precioso el caso de Francisco el Youtuber. Y cómo en sesión la madre y el hijo se han constituido como equipo para vencer al enfado y la impulsividad.

C-Y hemos visto como eso surge de una conversación que no sigue los mapas, que sigue lo que el chico está creando en la consulta. No hay técnica, hay una forma de preguntar coherente con la cosmovisión narrativa pero las preguntas surgen de la propia conversación. Y la creatividad fue de Francisco, no del terapeuta.

M- Ha surgido un tema que es habitual encontrarse en la consulta. El enfoque lúdico de la terapia narrativa propicia que cuando trabajamos con menores de edad inicialmente algunos padres desconfíen, que piensen que un enfoque "tan poco serio”, en el que sus hijos se divierten en la sesión y juegan con sus problemas, no sea suficiente, y que pidan "más caña al chico" como en el caso que nos has contado. ¿Cómo prevenir eso?

C- No sé si podemos prevenirlo. La gente asocia terapia a problemas, a pasarlo mal... no suelen venir preparados para un enfoque lúdico. Pero sí podemos explicarles lo posibilitador que resulta ver al niño como un niño, tratarle como tal, comportarnos también como niños, dejar que la imaginación fluya... y eso a veces puede resolver ciertos problemas. Sugerirles que esperen a ver cómo sigue...

M-Y en este caso la madre finalmente pudo verlo bien claro. Muy emocionante el fragmento en el que el hijo reivindica el equipo en lugar del enfado y pide a la madre que se posicione y ella lo hace. El fragmento de conversación doméstica que te traen grabado.

C-Sí, cuando el hijo le dice a la madre: Yo prefiero el equipo, no quiero que vuelva a aparecer el enfado (...) Yo prefiero el equipo, ¿y tú? Sólo dime: sí o no. Y la madre responde convencida: Yo quiero al equipo. Y siguen hablando sobre cómo mantener el equipo. Se dio la magia.

M- Me gusta también la idea de hacernos ver el caso desde la comunidad que está intentando ayudar a Francisco. Pero también cómo Francisco y su familia contribuyen a que la comunidad narrativa mejore. La bidireccionalidad que se da. 
Quiero decir, desde la terapia narrativa movilizamos al entorno de Francisco para que formen parte de la creación y/ o validación de la historia alternativa que está en la base de su cambio. Los profesionales con los que tú compartes su historia también podemos dar mensajes de reconocimiento que tú compartes con la familia para ayudarles a mejorar. Pero ellos, compartiendo su historia, también ayudan a que la comunidad narrativa mejore. Eso es muy potente, y muy congruente con los fundamentos de la terapia narrativa. Cuando le dices a la madre "cuéntale (al hijo) para que quede para todas las familias del mundo cómo hacer un equipo" (y grabarlo, como en este caso, o escribirlo en un libro de noticias para futuros consultantes u organizar un pequeño evento con entrega de diplomas...) ahí estás validando la narrativa alternativa, sustentándola en la comunidad que va a poder contribuir a que permanezca.

C- Es trabajo comunitario. Se trata también de co-construir nuestras disciplinas. ¿Cómo podemos hacerlo? Ya sea en colegios, en comunidades en situación de riesgo, menores, ... Se construye hablando, dialogando, haciendo presentaciones, mandándole cartas a nuestros consultantes, recibiendo cartas de nuestros consultantes... escuchándolos, invitándoles a los congresos, conferencias, talleres... para que vengan y expresen sus opiniones. Porque no sólo los profesionales enseñamos, también los consultantes nos enseñan y mucho. Es un camino de ida y vuelta. Es una comunidad. Y por eso se creó AETEN. La Asociación Española de Terapia Narrativa es una comunidad, un conjunto de gente a quienes les interesa hacer este tipo de cosas. Es una comunidad que se mueve para empezar a repensar la competitividad y la desigualdad. Desde la terapia narrativa la igualdad se lleva a la práctica, nos alejamos de la competitividad que hay entre disciplinas, entre escuelas...

M- El sentimiento de comunidad es potente, se lleva a la práctica, y la "divulgación de las noticias” es parte importante de ello. Freedman y Combs (1996) reflexionaban y siguen haciéndolo en trabajos posteriores (e.g., Freeman, Epston y Lobovits, 2001) sobre cómo en la cultura dominante la terapia se ve como un trabajo que debe mantenerse en secreto y en la subcultura narrativa esto es justamente lo contrario. Cuando el trabajo se centra en construir una historia preferida, un yo preferido, al fin y al cabo, la persona no necesita ocultar, más bien al contrario, puede enorgullecerse de mostrar su yo preferido y darle todavía más consistencia haciéndolo evidente en su entorno. La terapia narrativa desde su origen hace esto mediante cartas, reuniones, certificados, rituales de celebración del cambio.... Todo ello presencial, como eran las cosas en los 80-90. ¿Qué transformación crees que está sufriendo todo esto en el mundo de las redes sociales, los emails, las comunidades virtuales...?

C- Todavía en algunos casos podemos hacer campañas de cartas (Madigan, 2019) y la gente se sorprende al recibir cartas en el buzón, ijajaja! Yo, por lo general, trabajo con el email y con el whatsapp. Esto último con adolescentes que se cansan de leer una carta o un email extenso. Serían mensajes directos, con preguntas muy específicas y, por supuesto, cortos. Todavía tengo que investigar si causan el mismo 
efecto que un email más extenso.

M- Nos hablabas también de la terapia como arte, diferenciando el arte de la artesanía hecha de manera repetitiva. Me gusta la reivindicación de la terapia como arte. Es valiente y suena casi irreverente plantearla en un momento como el actual, en el que la comunidad dominante está preocupada justamente por la reivindicación de la psicoterapia como ciencia, a la caza de las denominadas pseudo-ciencias. ¿Cómo crees que se posiciona la terapia narrativa en este debate? ¿se puede ser a la vez arte, hacer magia... y ciencia?

C- Uno de los seminarios que más le gusta hacer a David Epston en entornos hispano hablantes, tiene que ver con las preguntas con "duende" (tomando la palabra de Federico García Lorca). Tiene que ver con analizar por un grupo de terapeutas aquellas preguntas que más les tocaron, conmovieron o sorprendieron por lo inusual, poético, transformador, de las mismas. La investigación cualitativa y etnográfica, nos da muchas herramientas para poder seguir haciendo "magia científica", si se me permite la expresión. Por otro lado, hay muchas investigaciones, e irán surgiendo más, que desde lo cuantitativo (discurso hegemónico en las ciencias sociales en la actualidad) muestran lo valioso de las prácticas narrativas. De todos modos, aunque se discurra en paralelo, la magia de la terapia narrativa tiene que ser preservada. En este sentido, es importante tener en cuenta la diferencia entre artesanía y arte. Artesanía pueden ser cosas que se hacen de manera repetitiva, una y otra vez. El arte es algo diferente. En cada conversación salen nuevas cosas o nuevas posibilidades. La pregunta es: ¿̇me tengo que adherir a los mapas o puedo empezar a hacer mis propios mapas?, ¿puedo alejarme un poco de lo que plantean Michael White y David Epston y crear mis propias formas de hacer consulta, mis propias formas de conversar? La respuesta debiera ser afirmativa y no siempre repetir o replicar... ¿Eso quiere decir que anulamos el tema de los mapas o que ya no hay formarse? Claro que no. Es necesario que aprendamos a tocar los acordes y las escalas para después improvisar. Claro que tengo que aprender ciertas cosas. Luego tengo que interiorizarlas, hacerlas mías, y después ya se puede improvisar. Es un proceso. Es práctica, práctica y más práctica. Necesito aprender sobre los tipos de conversaciones, qué es lo que hay por detrás de la terapia narrativa. Pero una vez que lo sé... me olvido e improviso. Haz arte, no hagas artesanía. Eso es el espíritu de aventura de la terapia narrativa.

De alguna forma el espíritu de la aventura narrativa y la forma de co-construir el futuro de nuestras disciplinas parte de no limitarnos ni en libertad ni en imaginación replicando modelos de otros. No hace falta tampoco que creemos uno nuestro, que lo escribamos en libros. Simplemente es que tú hagas tus propias actividades, te acerques a las personas con respeto, crees conversaciones de forma diferente y eso va a enriquecer muchísimo lo que es la terapia narrativa. 


\section{Referencias bibliográficas}

Freedman, J. y Combs, G. (1996). Narrative therapy: the social construction of preferred realities. Nueva York, NY: Norton.

Freeman, J., Epston, D. y Lobovits, D. (2001). Terapia narrativa para niños. Barcelona, España: Paidós (Trabajo original publicado en 1997).

Madigan, S. (2019). Narrative Therapy. Washington, DC: American Psychological Association.

O’Hanlon, B. (1994). The third wave. Family therapy networker, 18(6), 18- 29.

White, M. (1984). Pseudo-encopresis: from avalanche to victory, from vicious to virtuous cycles. Family Systems Medicine,2(2), 150-160. https://doi.org/10.1037/h0091651

White, M. (1997). Guías para una terapia familiar sistémica. Barcelona, España: Gedisa. 